\title{
Development Prospect and Dilemma of Social Work under the Big Data Heting Sun
}

School of Politics and Administration, Wuhan University of Technology, Wuhan, 430063, China; 569542267@qq.com

Keyword: Big Data, Social Work

\begin{abstract}
The development of Internet technology has gradually shaped the brand new form of life, and the big data technology as its most by-product has provided the growth of social work with the new opportunities by collecting the massive information rapidly before analyzing and feeding back in an efficient and scientific way. It is waking up to the fact that the social work is confronted with the difficulties and challenges in the context of big data while making the best of its characteristics to transmit the professional social services. The combination of both sides shall be treated in a dialectical way, thus increasingly enhancing the influence of the whole discipline.
\end{abstract}

\section{Introduction :}

It is demonstrated in the fortieth Statistical Report on the Development of China's Internet issued by China Internet Network Information Center in Beijing that there have been 751 million netizens in China as of June 2017, accounting for one fifth of the total Internet users worldwide. And China's Internet penetration rate of $54.3 \%$ reaches 4.6 percentage points of the global average level. Building the new form for the social life, the Internet is making the influences on the entire society as never before. And the big data which is perceived as the by-product of the sharply progressing Internet at present serves to store all aspects of people's daily life including social activities, commercial trade and life ranges in the form of data, thus making an analysis, handling and finding out the existing law and commonness. At the beginning of its generation, it was distinguished by Volume, Velocity, Variety, Value and Veracity. During the process of greatly enhancing our understanding about how expansive the world is and what the universe is all about, it is changing our life, work and the way of thinking dramatically and affecting the basic concepts, research logic, research methods and technology of social sciences. And opportunities are up for grabs for the research and practice of social work, accompanying with challenges.

As the discipline to design the solutions to the problems based on the analyzed needs of the service object through the investigation, the social work shall never get around the opportunities and challenges brought by the big data in the face of the limitations existing in the traditional questionnaire or interview survey, including one sidedness and low efficiency. It is expected to make the best of the characteristics of big data for more efficient transmission of professional social services and to advocate reasonable social policies more accurately, so as to realize the goals of the profession.

\section{The Necessity of Introducing Big Data into Social Work}

\subsection{More scientific data is required by the empirical-based social work.}

Before conducting the social work practice, it shall be based on the objective and scientific evidence, and its intervention behavior on the case subject is consequently targeted and scientific. However, the absence of the effective data collection and analysis system in the current social work service system triggers to the greatly obstructed data integration and analysis on the client requirements. At the same time, the questionnaires and interviews as the methods to collect data in the traditional social work make it unlikely to sample and investigate on a large scale because of the restricted time and funds. In spite of the certain pertinence possessed by the designed problems based on the requirements, the small scale as the inherent disadvantage will definitely lead to the impossibilities 
of considering all aspects during the process of conducting the social work practice. And the generation of big data can effectively improve this situation. Under the circumstance of the big data, it is more accessible to the information. Apart from the advantages like rapid collection, large quantity and strong authenticity, its prompt and scientific analysis of massive data can reflect the common characteristics and needs of users as a whole, making it possible to conduct the social work more rapidly and scientifically.

\subsection{The localization of social work shall be supported by big data.}

Originated from the West, social work is guided by the western theoretical methods after its introduction into China, and the human relationship society with Chinese characteristics as well as community environment make it unlikely to develop the social work under the guidance of the traditional theory. However, the social environment under the big data plays a role in objectively analyzing the people's participation, identification and effectiveness in the social activities. Moreover, it can also find out the rules and correlation of service objects at large, in a bid to point out the problems and shortcomings in the activities conducted by the local social workers as well as provide the scientific data guidance for the localization development of social work.

\subsection{Interdisciplinary cooperation and dialogue are necessary for social work.}

As the profession serving to address the social problems, promote the social harmony and accelerate the social progress, the social work shall keep pace with the times in the development and learn the different problems existing in different stages, thus keep improving the knowledge theory system. In this regard, it is the inevitable course for the social work to seek for cooperation and dialogue with other disciplines. The researches on the social work are provided with the new paths by the rising big data, further supporting collaboration among different domains methodically.

To sum up, the combination of social work and big data technology can enhance the professionalism and scientific nature of social work. In the traditional social work pattern, the hysteretic and small-scale information data acquisition and analysis lead to the sluggishness of institutions embarking on social work when intervening in the certain social problems. The applicable big data technology works to detect social problems in a timely manner before responding quickly, so as to overwhelm the difficulties, escape from the limitations of traditional investigation methods and samples and study the social work intervention more widely and rapidly.

\section{The Application of Big Data in the Development of Social Work}

At present, the researches on the big data and social work are still at the starting stage, and the practical application is merely the experimental development to some extent. But the author believes that the social work under the big data can be more broadly applicable. Accordingly, the following four aspects will be elaborated in the paper.

\subsection{To provide scientific evidence basis for social work}

The big data plays a role in obtaining and updating the authentic human behavior data in real-time manner, which can avoid the dependence on subjective reports in the process of data collection and effectively explain or reveal the continuous change trend of group psychological characteristics to control the pulse of society. The development of the Internet makes the network platform become one of the most channels for people to express their opinions and interests. And the network public opinion, which has turned into the main form of public opinion, has provided new opportunities for the timely determination of policy formulation and service orientation after the timely and effective perception of social psychological situation and analysis of network public opinion as well as the determined public demands. To a certain extent, the obstacles existing in the traditional questionnaire survey in a wide range, including long period, high cost, failure in updating timely, low ecological effect and difficult implementation of longitudinal tracking, have been eliminated. Additionally, the instantaneously updated big data can make the real-time assessment on the 
effectiveness of social work intervention, conducive to the improvement and development of follow-up intervention activities.

\subsection{To provide efficient technical means for crisis intervention}

The assessment and crisis intervention data of the risk population have been provided with the technical means which are efficient and accurate by the big data. With the help of the social media big data like Micro-blog, it can perceive the emotional changes and mental health of users and take the suicide prevention in an effective way. And the user big data like Baidu can identify the distribution and high-risk areas of the disease before taking the targeted prevention and control. Meanwhile, the adoption of the Micro-blog data in the group event risk prediction can sound a warning to the critical state of group social attitudes, find out and identify the factors affecting the social stability and provide the data support for timely resolving the potential mass incidents, thus providing a strong tailwind for the social management innovation. In addition, the Internet and big data technology can be used timely for the dissemination of emergency information in the event of crisis, and it can effectively implement the early warning system and assist the rescue work.

\subsection{To provide evidence for the formulation of public policy}

The analysis of the distribution of public service facilities and the accessibility to the relative service population will help to discover the demands of traffic and facilities in time, so as to enhance the fairness of public service resources. For example, the previous data which are inaccessible or ignored will also play an important role in promoting the residents' health equity along with the constantly disclosed medical big data. Taking the case for the research on the poverty, it is part of the long-term dilemmas confronted by the human society development in the twenty-first Century, and is also the important services field of social work. For the developing countries, the key to precisely formulate the strategies for poverty eradication is to estimate the regional poverty levels. And the existing studies have shown that nighttime comprehensive data from the remote sensing satellites can serve as a useful tool for assessing poverty at the county level in China, and the effectiveness of nighttime light big data in predicting poverty is also confirmed by the similar studies. Moreover, the mobile phone big data are used for poverty prediction. It can be seen that the big data has become an important means of accurate positioning of people and regions in straitened circumstances. For the social workers, the precise poverty alleviation work can be helped by the technology.

\section{Challenges Confronted by Social Work in Big Data Environment}

The challenges that may arise in the application process cannot be ignored while the big data brings new opportunities for social work research, including data security and usage ethics, data processing technology and research methods.

\subsection{How to ensure the data application in the safe and ethical manner?}

Data security and usage ethics are perceived as the central issues, and personal information is constantly being produced accompanying with the popularizing information and communication technology, related electronic equipment and application program in people's daily life. During the process of helping the researches in effectively identifying and depicting the specific individuals and groups, the user's privacy is completely exposed. In practice, it is the key to protect user's privacy and establish the standard system for data usage ethics. And the digital technology and big data analysis means shall be introduced in the service planning and policy making of social work, to set up the strict framework to balance privacy protection and public interests.

\subsection{How to provide technology for effective data processing?}

In terms of the massive quantity of information data which is complicated and fragmented, it is a core topic about how to quickly clean and process it, extract effective and targeted information and prevent the information from being lost in the process of filtering. The big data usually consists of 
all data from the specific source, with most of them making no sense. And the frequently contained incomplete information and wrong data shall be processed effectively in the stage of data analysis. The failure in meticulously reviewing and planning the big data which is complex and highly variable will leads to the potential misunderstanding and errors. Apart from the structured data that is commonly used, the big data is also widely distributed in the form of unstructured text, images and recording. In this regard, the conventional methods of classifying, managing, processing, storing and analyzing data are not enough to obtain full value from big data.

\subsection{Debate on research methods}

The perception and interpretation of personal experience shall not be ignored while stressing the big data. In the context of big data, what role shall big data play in the process of research and knowledge generation? The author is convinced that we shall not merely insist on the data accuracy and actively extract the effective information in the course of integrating both sides, but also shall realize that the data purely serving as the tool for theory is to prove the presupposition and hypothesis of the theory. The scenarios, emotions, and realities behind the data should also be considered, and it shall attach great importance to the docking between the offline data and relevant theoretical analysis in the face of analysis result. To a certain extent, the big data indeed provides with the way to make discipline research more objective, but the certain subjective consciousness and deviation appear in the data selection and extraction. And we shall be vigilant against the contrarieties between increased big data quantitative research and qualitative study.

We are not supposed to abandon the traditional paradigm of small data research while the big data brings a lot of opportunities for social work research and data-oriented research. There are also problems of sample bias and representativeness in the big data, and the approaches and means of data processing can effectively reduce the sample deviation. We shall persist in the standard setting of researched problems, the control of sampling samples and the strict standards of data acquisition and analysis process. In the actual practice, it is expected to be based on the overall trend grasped by the big data and the specific details captured by the small data.

\section{Conclusion}

The brand new opportunities and challenges are brought by the big data in the development of social work, and it is absolutely likely to realize the professional goals of social work with the help of big data, which is a broad field of exploration. On the one hand, however, we shall attach great importance to the value of big data in the social work research and practice as well as keep exploration, practice and innovation owing to the impact brought by the big data. On the other hand, we shall also reflect on the application of big data calmly, take the potential crises and risks into considerations, rethink the way to promote the development of social work itself under this circumstance and enhance the influence of the whole discipline.

\section{References}

[1]. P. Khan,L. Dominelli. The impact of globalization on social work in the UK[J] . European Journal of Social Work . 2000 (2)

[2].Wanzhen Chen.Big Data-Driven Social Work: Prospects and Challenges [J]. Social Sciences, 2017, (07): 74-81.

[3].Xiaobin.Peng.Mechanism and Path of Social Work Involved in the Prevention of Environmental Mass Incidents - Based on Big Data [J]. Social Work. 2016,(04):62-71+127.

[4]. Mao Lishu.Comparative study of the social work services from the perspective of glocalization-Chinese and Foreign physically disabled children's center as an example [D].Central China Normal University.2016 